\title{
Monitoring and Evaluation Mechanism for Multi- Center Capacity Building Gestational Diabetes Program for Physicians in India
}

\author{
Megha Sharma* \\ Centre for Control of Chronic Conditions / Training, Public Health Foundation of India, Delhi, India
}

\section{Objective}

With implementation of program on all India level aim is to develop a robust monitoring and evaluation system to ensure quality assurance and standardized course delivery on all India level.

\section{Introduction}

International Diabetes Federation (IDF) estimates that 21.4 million women in 2013 had some form of hyperglycaemia in pregnancy and in India alone an estimated 4 million women have GDM. Recognizing the shortfall of trained manpower; Certificate Course in Gestational Diabetes Mellitus (CCGDM) was launched in 2012; since then it has spread across 17 states and 39 cities across 55 regional training centers and trained 2400 Primary care physicians (PCP) all across India.

\section{Methods}

Inadequate knowledge among PCPs is one of the major obstacles in the prevention and management of GDM therefore we developed a comprehensive 4 modular course to train PCPs which is a joint program of Public Health Foundation of India and Dr. Mohan's Diabetes Education Academy(WHO Collaborating centre) with final approval of the course curriculum by national expert panel.A cadre of Observers who are eminent Public Health Professionals were the backbone. It incorporated on-site monitoring visits every month to Regional Centers for evaluation on the basis of standardized indicators and formats. Baseline survey was employed to assess Knowledge Attitude Practices of GDM among participants,SMS Real Time E- Monitoring System was used for meticulous and timely follow up, End Line Evaluation was planned at the end of program; by the means of face to face interviews information was received on structure of curriculum, teaching methods, session environment, knowledge improvisation, value addition.

\section{Results}

The analysis of data generated was subsequently published in reports and shared with stakeholders. The impeccable delivery of CCGDM has given it recognition from IDF and accreditation from South Asian Federation of Endocrine Society. The results of end line evaluation concluded with improved knowledge of PCPs (Pre-test \& Post-test score, $\mathrm{P}<0.001$ ), value addition to knowledge $(98.5 \%)$, value addition to skills $(96.8 \%)$, program enhanced routine treatment plan $(95 \%)$; ideal for learning $(96.1 \%)$, useful case studies (88.5\%); interactive and informative sessions (98\%), faculty competency (94\%); professional network and referral $(67.6 \%)$.

\section{Conclusions}

The launch of capacity building initiative is just the beginning but final success will depend on how effectively we monitor and evaluate it.

\section{Keywords}

Gestational Diabetes Mellitus; Monitoring \& Evaluation; Pan India

\section{Acknowledgments}

Prof. D. Prabhakaran, Dr. Sandeep Bhalla, Dr. V. Mohan, Dr. Ranjit Unnikrishnan

\section{References}

IDF Altas 6th edition

*Megha Sharma

E-mail: meghs211@yahoo.com 